\title{
Estudio etnobotánico y etnofarmacológico de plantas medicinales de Tambopata, Madre de Dios, Perú
}

\author{
Ethnobotanist and ethnopharmacological study of medicinal plants of \\ Tambopata - Madre de Dios, Perú
}

\author{
Yuly Molina Ayme*
}

http://dx.doi.org/10.21503/CienciayDesarrollo.2011.v14.01

\section{RESUMEN}

El proyecto general comprende tres etapas: el estudio etnobotánico y etnofarmacológico de las plantas medicinales en la región Madre de Dios, el estudio fitoquímico de las plantas más potencialmente activas, y finalmente la elaboración de productos derivados.

La primera etapa busca registrar la mayor información sobre lo etnobotánico y etnofarmacológico en la región, por ser esta la parte de la hoya hidrográfica del Madre de Dios (provincias de Tambopata, Manu y Tahuamanu) que conserva estas tradiciones muy antiguas sin alteración, gracias a que hasta la actualidad se ha mantenido casi enclaustrada, con escasa presencia foránea.

El presente estudio se realizó en la provincia de Tambopata, en el año 2011, y en el 2012 se continuará en las provincias de Manu y Tahuamanu. El trabajo se desarrolló teniendo en cuenta que somos herederos de un ancestral conocimiento indígena de nuestra región, el mismo que está en vías de perderse. Por eso nos vimos en la obligación académica de ser los pioneros en acudir al rescate de este conocimiento.

Para el efecto, contamos con el apoyo de connotados profesionales a nivel nacional, como el Dr. Oswaldo Salaverry, Director del Centro Nacional de Medicina Intercultural del Ministerio de Salud, y el Dr. Alfredo Tupayachi, investigador asociado al Herbario Vargas (CUZ), en Cusco.

La variada y compleja geografía del Perú ha dado lugar a una gran diversidad de climas, ecosistemas y formas de vida. En el Perú existen 84 de las 104 zonas de vida caracterizadas en el mundo, y posee 28 de los 32 climas descritos. Se han caracterizado 11 ecorregiones, que sitúan al Perú entre los países más ricos en formas de vida en el planeta, ubicándose en la lista de los diez países del mundo con mayor diversidad (Primack, 2002).

La importancia de este estudio radica en que busca establecer cómo la gente de una cultura y región hace uso de sus plantas nativas, a fin de coadyuvar a que este conocimiento se preserve en el tiempo. Pero además resulta importante también para la realización de estudios farmacológicos relacionados con plantas medicinales.

Palabras clave: etnobotánico, etnofarmacológico, fitoquímico, ecosistema.

* Químico farmacéutico, docente principal de la Escuela Académico-Profesional de Farmacia y Bioquímica de la UAP, Filial Puerto Maldonado. En colaboración con Atencio Paco, Cristina M.; Bocángel Farfán, Sherley; Chasin Flores, Yajhayda; Cóndor Quispe, Fabiola R.; Huesembe Cruz, Jhonny; Jara Cruz, Mónica S.; Macutela Quispe, Esperanza; Malatesta Aulestia, Silvia A.; Mallqui Fabián, Gaby S.; Meza López, Nancy; Monteagudo Polo, Jorge M.; Parrillo Mamani, David A.; Quispe Guillén, Eber; Quispe Mamani, Sonia R.; Quispesayhua Tuni, Genoveva E.; Torobeo Ramos, Jhossep S. (moayu77@hotmail.com) 


\section{ABSTRACT}

The overall project comprises three stages: ethnobotanical and ethnopharmacological study of medicinal plants in the region of Madre de Dios, the phytochemical study of potentially active plants and finally the development of products.

The first stage, sought to capture as much information about the ethnobotanical and ethnopharmacological in the region, as this is the part of the watershed of the Madre de Dios (provinces of Tambopata, Manu and Tahuamanu), which preserves these ancient traditions without be altered, because until now has remained virtually cloistered with little foreign presence.

This study is located in the province of Tambopata and has been made in the year 2011. In the year 2012 will continue in the provinces of Manu and Tahuamanu.

This work was developed taking into account that we are heirs of indigenous ancestral knowledge of our region and is on track to miss. We are therefore in the academic obligation to pioneer rescue knowledge.

This work has the support of nationally renowned professionals, Dr. Oswaldo Salaverry Director of the National Center for Intercultural Medicine of the Ministry of Health, Dr. Alfredo Tupayachi, and Vargas Herbarium Research Associate to CUZ. Cusco.

The varied and complex geography of Peru has led to a great diversity of climates, ecosystems and livelihoods. In Peru there are 84 areas of life, of the 104 characterized in the world and 28 of the 32 climates described. We have characterized 11 eco-regions, which lie in Peru between the richest countries in forms of life on the planet, reaching the top ten countries worldwide with more diversity. (Primack, 2002)

The importance of this study lies in the way that people of a culture and region make use of their native plants and that this knowledge is preserved in time. This information is also important to perform pharmacological studies related to medicinal plants.

Key words: ethno botanical, ethno pharmacological, phytochemical, ecosystem.

\section{INTRODUCCIÓN}

Los petroglifos en el río Shinkebeni (petroglifos de Pusharo) indican una muy antigua presencia de seres humanos. Se cree que los arahuacos (o sus antecesores) llegaron en migraciones y que de ellos se derivaron muchas etnias. Algunas tribus, como la machiguenga, sobreviven hasta nuestros días. Lo que hoy se conoce como Madre de Dios formaba parte del antiguo Imperio Inca, en la región conocida como Antisuyo. (Bermúdez, 2008.)

En nuestra región se presentan diferentes ecosistemas en los que se desarrolla una gran variedad de plantas, variedad que a su vez se manifiesta en las múltiples aplicaciones tradicionales y terapéuticas conocidas (Primack, 2002). Esta diversidad origina la necesidad de recabar información por zonas, aun tratándose de especies con el mismo nombre, pues en el ámbito local existe escasa información respecto de plantas medicinales de determinadas localidades (Camacho, 2005). Además, si tenemos en cuenta que algunas especies pueden tener el potencial para desarrollar nuevos medicamentos en el tratamiento de diversas enfermedades, este tipo de estudio sería un primer eslabón. Es así que resulta de suma importancia la realización de un estudio que recopile los conocimientos acerca de las plantas medicinales, puesto que ello ayudará a que puedan ser estudiadas científicamente en todos sus aspectos. 
No existe una fuente confiable de uso de plantas medicinales que ayuden a preservar el conocimiento ancestral de Madre de Dios y que sea una guía de tratamiento preventivo natural alternativo de cabecera para todos los pobladores de Madre de Dios.

A partir de esta constatación, centramos nuestro estudio en la utilización de plantas medicinales y su contribución al tratamiento de diversas afecciones en la provincia de Tambopata, en Madre de Dios.

\section{Objetivo general}

- Realizar un estudio etnobotánico y etnofarmacológico de plantas medicinales en la provincia de Tambopata, Madre de Dios.

\section{Objetivos específicos}

- Identificar y clasificar taxonómicamente las especies vegetales recolectadas a través del Herbario Vargas (CUZ).

- Sistematizar la información de las plantas medicinales de acuerdo al uso terapéutico o afecciones terapéuticas, parte utilizada y forma de uso a partir del conocimiento de los pobladores.

- Elaborar una guía de plantas medicinales de la región Madre de Dios.

\section{Conceptos importantes}

Medicina tradicional. Es la suma de todos los conocimientos teóricos, habilidades y prácticas basados en teorías, creencias y experiencias inherentes a las diferentes culturas, ya sean explicables o no, que se utilizan en el mantenimiento y conservación de la salud, así como en la prevención, diagnóstico y tratamiento de la enfermedad. Están basados exclusivamente en la experiencia y la observación, y son transmitidos verbalmente o por escrito de una generación a otra (CMP, 2006) (OMS, 1978).

Medicina complementaria. Se dice de la medicina "alternativa", "no convencional" o "popular", términos que se utilizan para referirse a muchos tipos de atención de salud no convencional que entrañan distintos grados de formación y eficacia (OMS, 2003).

Plantas medicinales. Son todas aquellas plantas que contienen uno o más principios activos, los cuales, administrados en la dosis adecuada, producen un efecto curativo frente a las enfermedades del hombre y de los animales. El hecho de contener más de un principio activo hace que una planta medicinal pueda servir para tratar diferentes afecciones o trastornos (OMS, 2002).

Etnobotánica. Trata del estudio de las relaciones existentes entre los vegetales y la especie humana. Por un sesgo metodológico y conceptual, desde su origen, la etnobotánica se ha centrado en los pueblos indígenas, las sociedades iletradas. Sin embargo, se ha demostrado repetidas veces que el conocimiento y práctica popular referidos a las plantas pueden ser también investigados en las sociedades más complejas.

Etnofarmacología. Es definida como la exploración científica interdisciplinaria de los agentes biológicamente activos y tradicionalmente empleados o conocidos por el hombre (Rivera, 2006).

\section{MATERIALES Y MÉTODOS}

\section{Tipo de estudio}

Es un estudio etnobotanico y etnofarmacológico de plantas medicinales de la provincia de Tambopata, Madre de Dios, y es del tipo transversal y descriptivo. Es descriptivo porque se describió la utilización de plantas 
medicinales por pobladores, y es transversal porque se estudia de forma simultánea en un momento dado.

\section{Muestra}

Tambopata cuenta con una población total de 78523 pobladores mayores de 18 años, según el Censo Nacional 2007 (INEI, 2008). La muestra utilizada fue de 299 pobladores, y la fórmula utilizada fue la siguiente:

$$
n=\frac{Z^{2} \times P(1-P) N}{E^{2}(N-1)+Z^{2} \times P(1-P)}
$$

\section{Donde:}

$\mathrm{N}$ : Población total.

Z: Confiabilidad.

P: Probabilidad de éxito en un $94 \%$ de la población.

E: Error.

n: Población maestral.
Los pobladores fueron muestreados por el método no probabilístico del tipo cuotas, ya que los pobladores tienen que cumplir con todos los criterios de inclusión.

\section{Criterios de inclusión}

- Para las entrevistas se incluyeron a veinte connotados curanderos de la zona, quienes nos dieron información sobre las plantas utilizadas.

- Para las encuestas se incluyeron a personas oriundas o que hubieran radicado en Madre de Dios por no menos de 5 años, para convalidar la información dada por los curanderos.

\section{Criterios de exclusión}

- Información de los pobladores que no pertenecían a la zona de estudio.

- Información de los pobladores que no tenían conocimiento de las especies vegetales de interés medicinal.

- Información de los pobladores menores de edad.

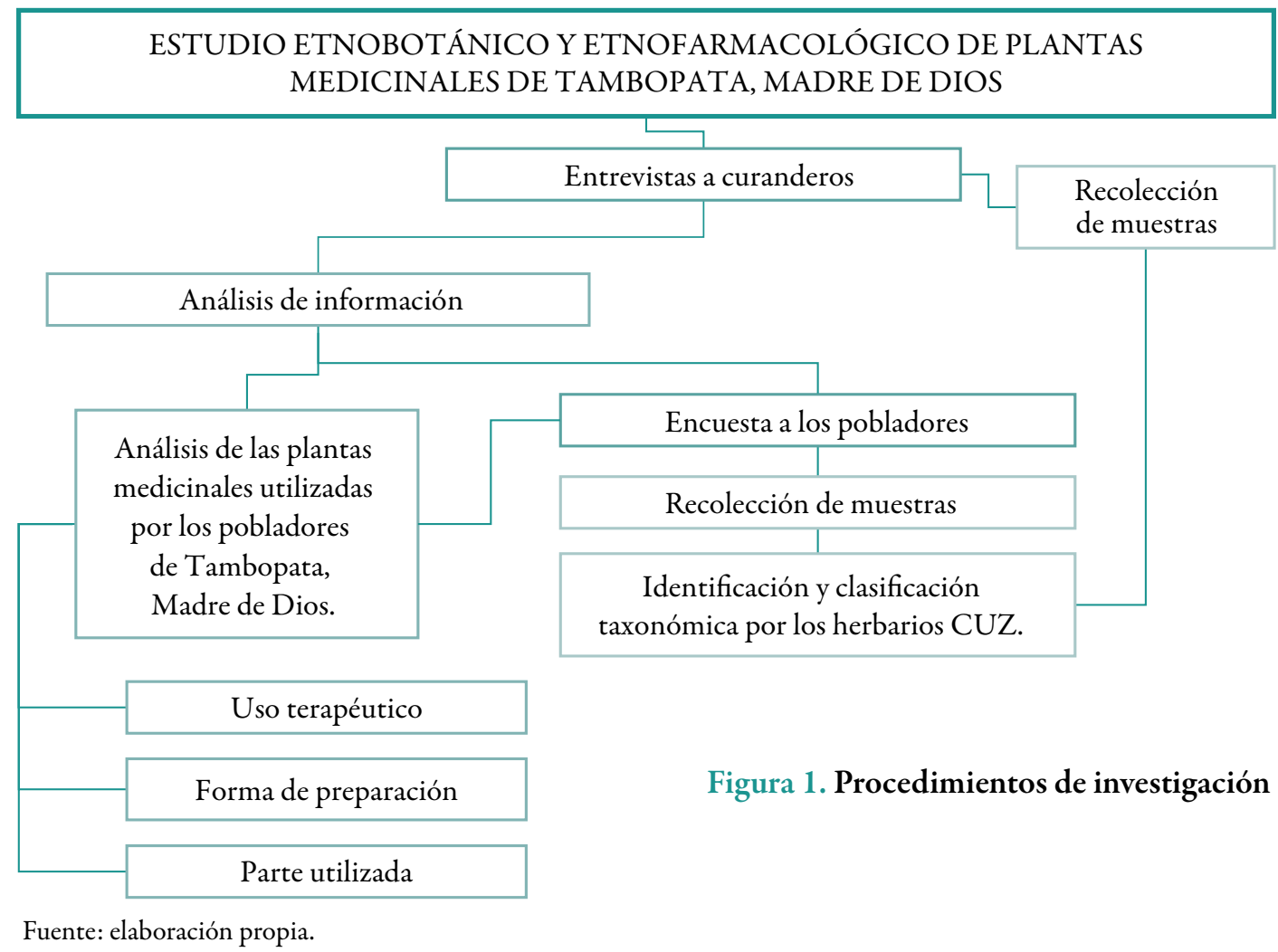


- Se realizó un reconocimiento de toda el área de la zona de estudio.

- Se realizaron salidas de campo en la provincia de Tambopata para desarrollar las entrevistas, las mismas que comprendieron a veinte pobladores (curanderos y pobladores conocedores de plantas medicinales). Así se obtuvo información sobre la ubicación, usos terapéuticos, forma de preparación, etc. (ANEXO No 01: Ficha de entrevista sobre la utilización de plantas). Para este procedimiento se contó con el apoyo de una grabadora, y paralelamente se fueron recolectando muestras botánicas por triplicado para su posterior armado, secado, herborización y su respectiva identificación en el herbario CUZ.
- Posteriormente, se realizaron las encuestas a los pobladores para validar la información obtenida en las entrevistas a los curanderos.

- Se utilizó el programa Excel para organizar toda la información según el uso terapéutico o las afecciones tratadas, formas de preparación y partes utilizadas de las especies medicinales.

Para validar los datos obtenidos a través de nuestras entrevistas y encuestas, se consultaron a dos profesionales: el antropólogo social Víctor Palomino Muñoz y la bióloga especialista en flora Alicia Cuba Villena, quienes, además de opinar sobre el contenido textual de nuestras herramientas de trabajo (ANEXOS $01 \mathrm{y}$ 02), discutieron el contenido de nuestros documentos según su especialidad, hasta llegar a una aprobación consensuada.

\section{RESULTADOS}

Tabla 1. Usos terapéuticos de cada especie vegetal de la provincia de Tambopata, Madre de Dios

\begin{tabular}{|c|c|c|c|c|}
\hline $\mathrm{N}^{\circ}$ & NOMBRE CIENTÍFICO & NOMBRE COMÚN & USO TERAPÉUTICO & CANTIDAD \\
\hline 01 & Abutilon sp. & Kirosillo & $\begin{array}{l}\text { Hepatoprotector, diurético, inflamación } \\
\text { renal. }\end{array}$ & 3 \\
\hline 02 & $\begin{array}{l}\text { Acmeltald ciliata (H.B.K.) } \\
\text { Cass. }\end{array}$ & Anastasia & $\begin{array}{l}\text { Anestésico dental, caries, analgésico dental, } \\
\text { espasmos estomacales, resfríos. }\end{array}$ & 5 \\
\hline 03 & Ageratina conyzoides $\mathrm{L}$. & Mankapaqui & $\begin{array}{l}\text { Disenterías fuertes, espasmos estomacales, } \\
\text { digestivo. }\end{array}$ & 4 \\
\hline 04 & Annona squamosa & Anona & Cáncer, raquitismo, diabetes & 3 \\
\hline 05 & Anoda hastata & Mora & Antidepresivo, diurético. & 2 \\
\hline 06 & $\begin{array}{l}\text { Artocarpus altilis } \\
\text { (Parkinson) Fosberg }\end{array}$ & Pan de árbol & $\begin{array}{l}\text { Fracturas, quemaduras, picaduras } \\
\text { de insectos, cáncer, hernia, anemia, } \\
\text { hipertensión. }\end{array}$ & 7 \\
\hline 07 & Begonia arbórea & Sello sello & Fiebre amarrilla. & 1 \\
\hline 08 & Bidens squearrosa & Pirka & $\begin{array}{l}\text { Infecciones estomacales, infecciones } \\
\text { urinarias, diurético, aftas, micosis, } \\
\text { adelgazante. }\end{array}$ & 6 \\
\hline 09 & Bixa urucurana Wild & Achiote & $\begin{array}{l}\text { Próstata, quemaduras, infecciones urinarias, } \\
\text { repelente, digestivo. }\end{array}$ & 5 \\
\hline
\end{tabular}




\begin{tabular}{|c|c|c|c|c|}
\hline 10 & $\begin{array}{l}\text { Brugmansia sanguinea } \\
\text { (R\&P) Don. }\end{array}$ & Floripondio rosado & Mordeduras de serpiente. & 1 \\
\hline 11 & Carica papaya L. & Papaya & Estreñimiento & 1 \\
\hline 12 & $\begin{array}{l}\text { Castilleja arvensis Schtd. } \\
\text { Chan }\end{array}$ & Madre madre & Dolor de huesos. & 1 \\
\hline 13 & Cecropia spp. & Cetico & Picaduras de insectos, hemorragia. & 2 \\
\hline 14 & Cedrela odorata & Cedro & Antipirético, tos, flatulencia. & 3 \\
\hline 15 & Cestrum sp & Hierba de cáncer & Cicatrizante, cáncer, abscesos. & 3 \\
\hline 16 & $\begin{array}{l}\text { Chromolaena laevigata } \\
\text { (Lam.) R.M. King \& H. } \\
\text { Rob. Var. arenarium Baker }\end{array}$ & Chillca & Reumatismo, fracturas, luxaciones. & 3 \\
\hline 17 & Citrus aurantium & Toronja & Digestivo, hepatoprotector. & 2 \\
\hline 18 & Coffea arabica $\mathrm{L}$. & Café & Cardiotónico, carminativo, parto doloroso. & 3 \\
\hline 19 & Columnea ericae Mansf. & Yawarchonka & Golpes. & 1 \\
\hline 20 & Copaidera paupera. & Copaiba & $\begin{array}{l}\text { Cicatrizante, micosis, hemorroides, cólicos, } \\
\text { úlceras. }\end{array}$ & 5 \\
\hline 21 & Costus scaber Ruiz \& Pav. & Caña caña & $\begin{array}{l}\text { Hepatoprotector, fiebre amarilla, presión } \\
\text { alta. }\end{array}$ & 3 \\
\hline 22 & Cyathea multiflora Sm. & Sano sano & $\begin{array}{l}\text { Cicatrices, reumatismo, presión alta, hernia, } \\
\text { hepatoprotector. }\end{array}$ & 5 \\
\hline 23 & $\begin{array}{l}\text { Cymbopogon citratus (DC.) } \\
\text { Stapf. }\end{array}$ & Hierba luisa & Infecciones estomacales, digestivo. & 3 \\
\hline 24 & Cyperus luzulae & Corta corta & Hemorragia. & 1 \\
\hline 25 & Croton cf. lechleri Mull Arg. & Sangre de grado & $\begin{array}{l}\text { Cicatrices, fracturas, hematomas, úlceras, } \\
\text { leucorrea, hemorroides, tumores, }\end{array}$ & 7 \\
\hline 26 & $\begin{array}{l}\text { Dracontium plowmanii G.H. } \\
\text { Zhu \& Croat }\end{array}$ & Jergón sacha & $\begin{array}{l}\text { Mordeduras de serpiente, hernia, úlcera, } \\
\text { parkinson. }\end{array}$ & 4 \\
\hline 27 & Desmodium neomexicanum & Runa manayupa & Reumatismo. & 1 \\
\hline 28 & Ecliptasp. & Kosmello & Fiebre. & 1 \\
\hline 29 & Erythroxylon coca Lam. & Coca & Dolor estomacal, picaduras de insectos, tos. & 3 \\
\hline 30 & Ficus insipida Willd. & Oje blanco & $\begin{array}{l}\text { Malaria, mordeduras de animales, parásitos, } \\
\text { purgante, reumatismo, anemia. }\end{array}$ & 6 \\
\hline 31 & Ficus paraensis (Miq.) Miq & Matapalo & $\begin{array}{l}\text { Fracturas, golpes, reumatismo, descensos, } \\
\text { abscesos. }\end{array}$ & 5 \\
\hline 32 & Guadua weberbaueri Pilg. & Paca & Dolor del corazón, depresión. & 2 \\
\hline 33 & Hamelia axillaris & Arcosacha & Micosis, cicatrices. & 2 \\
\hline 34 & Heliocarpus americanus L. & Yausapancho & Infecciones renales. & 2 \\
\hline 35 & Heteropsis oblongifolia & Tamishi & Dolor de muelas, hemorragias. & 2 \\
\hline
\end{tabular}




\begin{tabular}{|c|c|c|c|c|}
\hline 36 & Hura crepitans $\mathrm{L}$. & Catahua & $\begin{array}{l}\text { Reumatismo, contusiones, micosis, } \\
\text { antiofídico, asma, abscesos. }\end{array}$ & 6 \\
\hline 37 & $\begin{array}{l}\text { Jacaranda copaid (Aubl) D. } \\
\text { DON }\end{array}$ & Achiachiwa & Diurético, infecciones urinarias. & 2 \\
\hline 38 & Lantana Camara L. & Ipururo amarrillo & $\begin{array}{l}\text { Disentería, herpes, reumatismo, cólicos } \\
\text { estomacales. }\end{array}$ & 4 \\
\hline 39 & Lippia alba (Mill.) N.E.Br. & Cidra & $\begin{array}{l}\text { Flatulencias, dolores menstruales, colerina, } \\
\text { infecciones respiratorias. }\end{array}$ & 4 \\
\hline 40 & Lobelia decurrens Cav. & Solimán & $\begin{array}{l}\text { Acné, cicatrizante, mal de viento, mal de } \\
\text { chuqui, artritis. }\end{array}$ & 5 \\
\hline 41 & Manihot esculenta. & Yuca rosada & Fiebre, quemaduras, anemia. & 3 \\
\hline 42 & Mikania guaco H.LB. & Guaco & Reumatismo, inflamación faríngea. & 2 \\
\hline 43 & Mimosa pudica L. & No me toques & Anticonceptiva. & 1 \\
\hline 44 & $\begin{array}{l}\text { Munnozia hastifolia } \\
\text { (Poepp.) H. Rob. \& Brettell }\end{array}$ & Kinsaycucho & Inflamación renal, reumatismo. & 2 \\
\hline 45 & Musa spp. & Plátano & $\begin{array}{l}\text { Fiebre, cáncer, infecciones respiratorias, } \\
\text { calambres. }\end{array}$ & 4 \\
\hline 46 & Passiflora coccinea & Granadilla de monte & Fiebre & 1 \\
\hline 47 & Persea americana & Palta & $\begin{array}{l}\text { Disentería, caída de cabello, reumatismo, } \\
\text { artritis. }\end{array}$ & 4 \\
\hline 48 & Petiveria alliacea $\mathrm{L}$. & Montiago & Mal de chuqui, mal de viento, artritis. & 3 \\
\hline 49 & Picramnia sellowii Planch & Ayapira & Reumatismo, cicatrices. & 2 \\
\hline 50 & Piper aduncum L. & Matico mate & $\begin{array}{l}\text { Fiebre, dolor de huesos, artritis, } \\
\text { hepatoprotector, reumatismo, gonorrea, } \\
\text { úlceras, herpes. }\end{array}$ & 8 \\
\hline 51 & Piperpeltatum & Santa maría & $\begin{array}{l}\text { Cicatrices de chupos, debilidad muscular, } \\
\text { antiemético. }\end{array}$ & 3 \\
\hline 52 & Piper reticulatum $\mathrm{L}$. & Moco moco & $\begin{array}{l}\text { Inflamación, reumatismo, anemia, dolor de } \\
\text { huesos. }\end{array}$ & 4 \\
\hline 53 & Phyllanthus niruni $\mathrm{L}$. & Chanca piedra & $\begin{array}{l}\text { Reumatismo, hepatoprotector, cicatrices, } \\
\text { litiasis, galactógeno. }\end{array}$ & 5 \\
\hline 54 & $\begin{array}{l}\text { Phyllanthus pseudoconami } \\
\text { M. Arg. }\end{array}$ & Barbajo & Abscesos dérmicos, infecciones vaginales. & 2 \\
\hline 55 & $\begin{array}{l}\text { Pseudadephantopus spiralis } \\
\text { (Less) cronq }\end{array}$ & Cuchicara & $\begin{array}{l}\text { Hepatoprotector, } \\
\text { estreñimiento, empacho. }\end{array}$ & 4 \\
\hline 56 & Psidium guajava L. & Guayaba blanca & $\begin{array}{l}\text { Flatulencia, hepatoprotector, calambres, } \\
\text { conjuntivitis, estreñimiento, odontalgias. }\end{array}$ & 6 \\
\hline 57 & $\begin{array}{l}\text { Psittacanthus corynophalus } \\
\text { Eichler. }\end{array}$ & Soltaquesolta & Inflamaciones, hematomas. & 2 \\
\hline
\end{tabular}




\begin{tabular}{|c|c|c|c|c|}
\hline 58 & $\begin{array}{l}\text { Polypodium decumanum } \\
\text { Willd. }\end{array}$ & Rabo del mono & Reumatismo. & 1 \\
\hline 59 & Pycnopons cf. sanguineus L. & Callampa & $\begin{array}{l}\text { Hemorragias postparto, hemorragias } \\
\text { pulmonares, úlceras, heridas sangrantes. }\end{array}$ & 4 \\
\hline 60 & Rubus urticifolius Poir & Kari kari macho & Inflamación por golpe, tos. & 2 \\
\hline 61 & Schkubria pinnatus & Piquipichana & $\begin{array}{l}\text { Cicatrizante, antianémico, antiséptico } \\
\text { vaginal, antiofídico. }\end{array}$ & 9 \\
\hline 62 & Sida rhombifolia L. & Ucuchachupa & $\begin{array}{lrr}\text { Chupos, } & \text { cicatrices, } & \text { contusiones, } \\
\text { inflamación, } & \text { fiebre, } & \text { hematomas, } \\
\text { reumatismo. } & & \end{array}$ & 7 \\
\hline 63 & Senecio sp. & Diente de león & Diabetes, hepatoprotector. & 2 \\
\hline 64 & Smilax febrifuga Kunth & Zarzaparrilla & $\begin{array}{l}\text { Lesiones dérmicas, inflamación, } \\
\text { reumatismo, cálculos renales, ITU, sífilis. }\end{array}$ & 6 \\
\hline 65 & Solanum sessiliflorum Dunal & Cocona & $\begin{array}{l}\text { Úlceras, diabetes, hepatitis, picaduras de } \\
\text { insectos. }\end{array}$ & 4 \\
\hline 66 & Spilantehes urens & Venapiticha & Hemorragias, cólicos menstruales. & 3 \\
\hline 67 & $\begin{array}{l}\text { Stachytarpheta cayennensis } \\
\text { (Rich.) Vahl }\end{array}$ & Yanawacta & Fiebre, diabetes. & 2 \\
\hline 68 & $\begin{array}{l}\text { Tephrosia sinapou (Buch.) } \\
\text { Chev. }\end{array}$ & Barbasco & Reumatismo, rasca rasca. & 2 \\
\hline 69 & $\begin{array}{l}\text { Tessaria integrifolia Ruiz \& } \\
\text { Pav. }\end{array}$ & Pájaro bobo & $\begin{array}{l}\text { Hígado, próstata, asma, reumatismo, dolor } \\
\text { de muelas. }\end{array}$ & 5 \\
\hline 70 & $\begin{array}{l}\text { Triplaris surinamensis } \\
\text { Cham. }\end{array}$ & Palo santo & Anemia. & 1 \\
\hline 71 & $\begin{array}{l}\text { Triumfetta abutiloides St. } \\
\text { Hil. }\end{array}$ & Rata rata & Fiebre, inflamación faríngea, diurético. & 3 \\
\hline 72 & $\begin{array}{l}\text { Uncaria guianensis (Aubl.) } \\
\text { J.F. Gmel }\end{array}$ & Uña de gato & $\begin{array}{l}\text { Reumatismo, úlcera, prostatitis, artritis, } \\
\text { infecciones urinarias, disentería. }\end{array}$ & 6 \\
\hline 73 & $\begin{array}{l}\text { Urera caracasana (Jacq.) } \\
\text { Gaudich. Ex Grises. }\end{array}$ & Ortiga brava & $\begin{array}{l}\text { Hemorragias postparto, infección de } \\
\text { ovarios, anemia, hemorroides, reumatismo, } \\
\text { purificadora de la sangre. }\end{array}$ & 6 \\
\hline 74 & Verbena hispeda & Verbena & Alteraciones mentales, psicosis, inflamación. & 3 \\
\hline 75 & Zingiber officinale & Jengibre & $\begin{array}{l}\text { Flatulencia, espasmos estomacales, } \\
\text { emitismo, tos, reumatismo, conjuntivitis, } \\
\text { anticonceptivo. }\end{array}$ & 7 \\
\hline
\end{tabular}

Fuente de información: entrevistas y encuestas a los pobladores de Tambopata, Madre de Dios. 


\section{Tabla 2. Afecciones más frecuentes tratadas con especies medicinales en la provincia de Tambopata, Madre de Dios.}

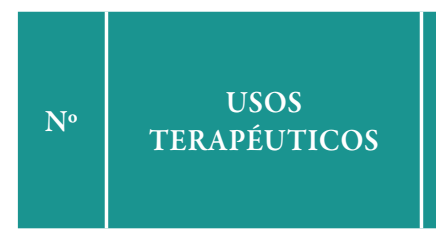

1 Reumatismo

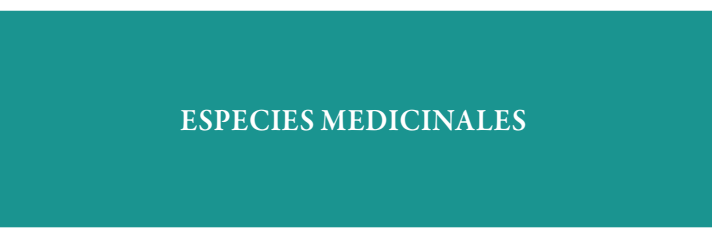

Jengibre, pájaro bobo, palta, piquipichana, rabo de mono, runa manayupa, sano sano, ayapira, barbasco, ortiga brava, catahua chanca piedra, chillca, guaco, ipururo amarillo, kinsaycucho, matapalo, matico mate, moco, oje blanco, ucuchachupa, uña de gato, zarzaparrilla.

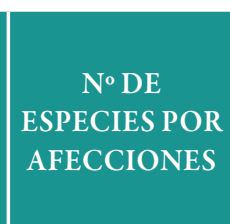

23
PORCENTAJE

SEGÚN LAS

ESPECIES

REGISTRADAS,

UTILIZADAS (\%)

9,23 2 Hepatoprotector Caña caña, chanca piedra, cuchicara, pájaro bobo,
diente de león, guayaba blanca, kirosillo, matico
mate, sano sano, toronja.

3 Antipirético

Cedro, granadilla de monte, kosmello, matico mate, plátano, rata rata, ucuchachupa, yanawacta, yuca rosada.

\begin{tabular}{|c|c|c|c|c|}
\hline 4 & Cicatrices & $\begin{array}{l}\text { Arcosacha, ayapira, chanca piedra, copaiba, hierba } \\
\text { de cáncer, sangre de grado, sano sano, solimán, } \\
\text { ucuchachupa. }\end{array}$ & 9 & 3,61 \\
\hline 5 & $\begin{array}{l}\text { Infecciones } \\
\text { respiratorias }\end{array}$ & $\begin{array}{l}\text { jengibre, cedro, cidra, coca, kari kari macho, } \\
\text { piquipichana, plátano. }\end{array}$ & 7 & 2,81 \\
\hline 6 & Úlceras & $\begin{array}{l}\text { Callampa, cocona, copiaba, jergón sacha, matico } \\
\text { mate, sangre de grado, uña de gato. }\end{array}$ & 7 & 2.81 \\
\hline 7 & Anemia & $\begin{array}{l}\text { Moco moco, oje blanco, ortiga brava, palo santo, } \\
\text { pan de árbol, yuca rosada. }\end{array}$ & 6 & 2,41 \\
\hline 8 & $\begin{array}{l}\text { Espasmos } \\
\text { estomacales }\end{array}$ & $\begin{array}{l}\text { Jengibre, anastasia, botoncillo, coca, copaiba, } \\
\text { ipururo amarrillo, mankapaqui. }\end{array}$ & 6 & 2,41 \\
\hline 9 & $\begin{array}{l}\text { Infecciones } \\
\text { urinarias }\end{array}$ & $\begin{array}{l}\text { Achiachiwa, ortiga brava, piquipichana, pirka, } \\
\text { uña de gato, zarzaparrilla. }\end{array}$ & 6 & 2,41 \\
\hline 10 & Diurético & Achiachiwa, kirosillo, mora, pirka, rata rata. & 5 & 2 \\
\hline 11 & Flatulencias & Jengibre, cedro, cidra, cuchicara, guayaba blanca. & 5 & 2 \\
\hline 12 & $\begin{array}{l}\text { Inflamación por } \\
\text { golpe }\end{array}$ & $\begin{array}{l}\text { Kari kari macho, soltaquesolta, ucuchachupa, } \\
\text { verbena, yawarchonka. }\end{array}$ & 5 & 2 \\
\hline 13 & Absceso dérmico & Barbajo, catahua, hierba de cáncer, matapalo. & 4 & 1,61 \\
\hline 14 & Analgésico dental & Anastasia, guayaba blanca, pájaro bobo, tamishi. & 4 & 1.61 \\
\hline 15 & Artritis & Matico mate, montiago, palta, solimán. & 4 & 1,61 \\
\hline 16 & Cáncer & Anona, hierba de cáncer, pan de árbol, plátano. & 4 & 1,61 \\
\hline 17 & Diabetes & Anona, cocona, diente de león, yanawacta. & 4 & 1,61 \\
\hline
\end{tabular}




\begin{tabular}{|c|c|c|c|c|}
\hline 18 & Digestivo & Achiote, hierba luisa, mankapaqui, toronja. & 4 & 1,61 \\
\hline 19 & Disentería & Ipururo amarrillo, mankapaqui, palta, uña de gato. & 4 & 1,61 \\
\hline 20 & Fiebre amarilla & Caña caña, oje blanco, piquipichana, sello sello. & 4 & 1,61 \\
\hline 21 & Fracturas & Chillca, matapalo, pan de árbol, sangre de grado. & 4 & 1,61 \\
\hline 22 & Hemorragias & Cetico, callampa, corta corta, tamishi, venapiticha. & 4 & 1,61 \\
\hline 23 & Hemorroides & $\begin{array}{l}\text { Copaiba, ortiga brava, piquipichana, } \\
\text { sangre de grado. }\end{array}$ & 4 & 1,61 \\
\hline 24 & Micosis & Arcosacha, catahua, copaiba, pirka. & 4 & 1,61 \\
\hline 25 & Picaduras de insectos & s Cetico, coca, cocona, pan de árbol. & 4 & 1,61 \\
\hline 26 & Próstata & Achiote, pájaro bobo, piquipichana, uña de gato. & 4 & 1,61 \\
\hline 27 & Anticonceptivo & Jengibre, no me toques, piquipichana. & 3 & 1,2 \\
\hline 28 & Contusiones & Catahua, matapalo, ucuchachupa. & 3 & 1,2 \\
\hline 29 & Dolor de huesos. & Madre madre, matico mate, moco moco. & 3 & 1,2 \\
\hline 30 & Hematomas & Sangre de grado, soltaquesolta, ucuchachupa. & 3 & 1,2 \\
\hline 31 & Hernias & Jergón sacha, pan de árbol, sano sano. & 3 & 1,2 \\
\hline 32 & Hipertensión & $\begin{array}{l}\text { Pan de árbol, piquipichana, sano sano, } \\
\text { caña caña. }\end{array}$ & 4 & 1,61 \\
\hline 33 & Inflamación renal & Kinsaycucho, kirosillo, zarzaparrilla. & 3 & 1,2 \\
\hline 34 & $\begin{array}{l}\text { Mordeduras de } \\
\text { serpiente }\end{array}$ & Floripondio rosado, jergón sacha, catahua. & 3 & 1,2 \\
\hline 35 & Litiasis & Chanca piedra, piquipichana, zarzaparrilla. & 3 & 1,2 \\
\hline 36 & Quemaduras & Achiote, pan de árbol, yuca rosada. & 3 & 1,2 \\
\hline 37 & Antidepresivo & Mora, paca. & 2 & 0,8 \\
\hline 38 & Antiemético & Santa María, jengibre. & 2 & 0,8 \\
\hline 39 & Asma & Catahua, pájaro bobo. & 2 & 0,8 \\
\hline 40 & Calambres & Guayaba blanca, plátano. & 2 & 0,8 \\
\hline 41 & Chupos & Santa María, ucuchachupa. & 2 & 0,8 \\
\hline 42 & Cólicos menstruales & Venapiticha, cidra. & 2 & 0,8 \\
\hline 43 & Conjuntivitis & Jengibre, guayaba blanca. & 2 & 0,8 \\
\hline 44 & Estreñimiento & Cuchicara, guayaba blanca. & 2 & 0,8 \\
\hline 45 & $\begin{array}{l}\text { Hemorragias } \\
\text { postparto }\end{array}$ & Callampa, ortiga brava. & 2 & 0,8 \\
\hline 46 & Herpes & Ipururo amarrillo, matico mate. & 2 & 0,8 \\
\hline 47 & $\begin{array}{l}\text { Infecciones } \\
\text { estomacales }\end{array}$ & Hierba luisa, pirka. & 2 & 0,8 \\
\hline
\end{tabular}




\begin{tabular}{|c|c|c|c|c|}
\hline 48 & Inflamación faríngea. & Guaco, rata rata. & 2 & 0,8 \\
\hline 49 & Mal de chuqui & Montiago, solimán. & 2 & 0,8 \\
\hline 50 & Mal de viento & Montiago, soliman. & 2 & 0,8 \\
\hline 51 & Resfríos & Anastasia. & 2 & 0,8 \\
\hline 52 & Acné & Solimán. & 1 & 0,4 \\
\hline 53 & Adelgazante & Pirka. & 1 & 0,4 \\
\hline 54 & Anestésico dental & Anastasia. & 1 & 0,4 \\
\hline 55 & Aftas & Pirka. & 1 & 0,4 \\
\hline 56 & Caída de cabello & Palta. & 1 & 0,4 \\
\hline 57 & Cardiotónico & Café. & 1 & 0,4 \\
\hline 58 & Caries & Anastasia. & 1 & 0,4 \\
\hline 59 & carminativo & Café. & 1 & 0.4 \\
\hline 60 & Colerina & Cidra. & 1 & 0,4 \\
\hline 61 & Debilidad muscular & Santa maría. & 1 & 0,4 \\
\hline 62 & Descensos vaginales & Matapalo. & 1 & 0,4 \\
\hline 63 & Empacho. & Cuchicara. & 1 & 0,4 \\
\hline 64 & Estreñimiento & Papaya. & 1 & 0,4 \\
\hline 65 & Galactógeno & Chanca piedra. & 1 & 0.4 \\
\hline 66 & Gonorrea & Matico mate. & 1 & 0,4 \\
\hline 67 & $\begin{array}{l}\text { Hemorragia } \\
\text { pulmonar }\end{array}$ & Callampa. & 1 & 0,4 \\
\hline 68 & Hepatitis & Cocona. & 1 & 0,4 \\
\hline 69 & Infección renal & Yausapancho. & 1 & 0,4 \\
\hline 70 & Infecciones vaginales & Barbajo. & 1 & 0,4 \\
\hline 71 & Leucorrea & Sangre de grado. & 1 & 0,4 \\
\hline 72 & $\begin{array}{l}\text { Alteraciones } \\
\text { mentales }\end{array}$ & Verbena. & 1 & 0,4 \\
\hline 73 & Luxaciones & Chillca. & 1 & 0,4 \\
\hline 74 & Mal del corazón & Paca. & 1 & 0,4 \\
\hline 75 & $\begin{array}{l}\text { Mordedura de } \\
\text { animales }\end{array}$ & Oje blanco. & 1 & 0,4 \\
\hline 76 & Parasitosis & Oje blanco. & 1 & 0,4 \\
\hline 77 & Parkinson & Jergón sacha. & 1 & 0,4 \\
\hline 78 & Parto doloroso & Café. & 1 & 0,4 \\
\hline 79 & Psicosis & Verbena. & 1 & 0,4 \\
\hline
\end{tabular}




\begin{tabular}{lllcc}
80 & Purgante & Oje blanco. & 1 & 0,4 \\
81 & Raquitismo & Anona. & 1 & 0,4 \\
82 & Rasca rasca & Barbasco. & 1 & 0,4 \\
83 & Repelente & Achiote. & 1 & 0,4 \\
84 & Sífilis & Zarzaparrilla. & 1 & 0,4 \\
85 & Tumor & Sangre de grado. & 1 & 0,4 \\
\hline & & $\mathbf{2 4 9}$ & $\mathbf{1 0 0}$ \\
\hline
\end{tabular}

Fuente de información: entrevistas y encuestas a los pobladores de Tambopata, Madre de Dios.

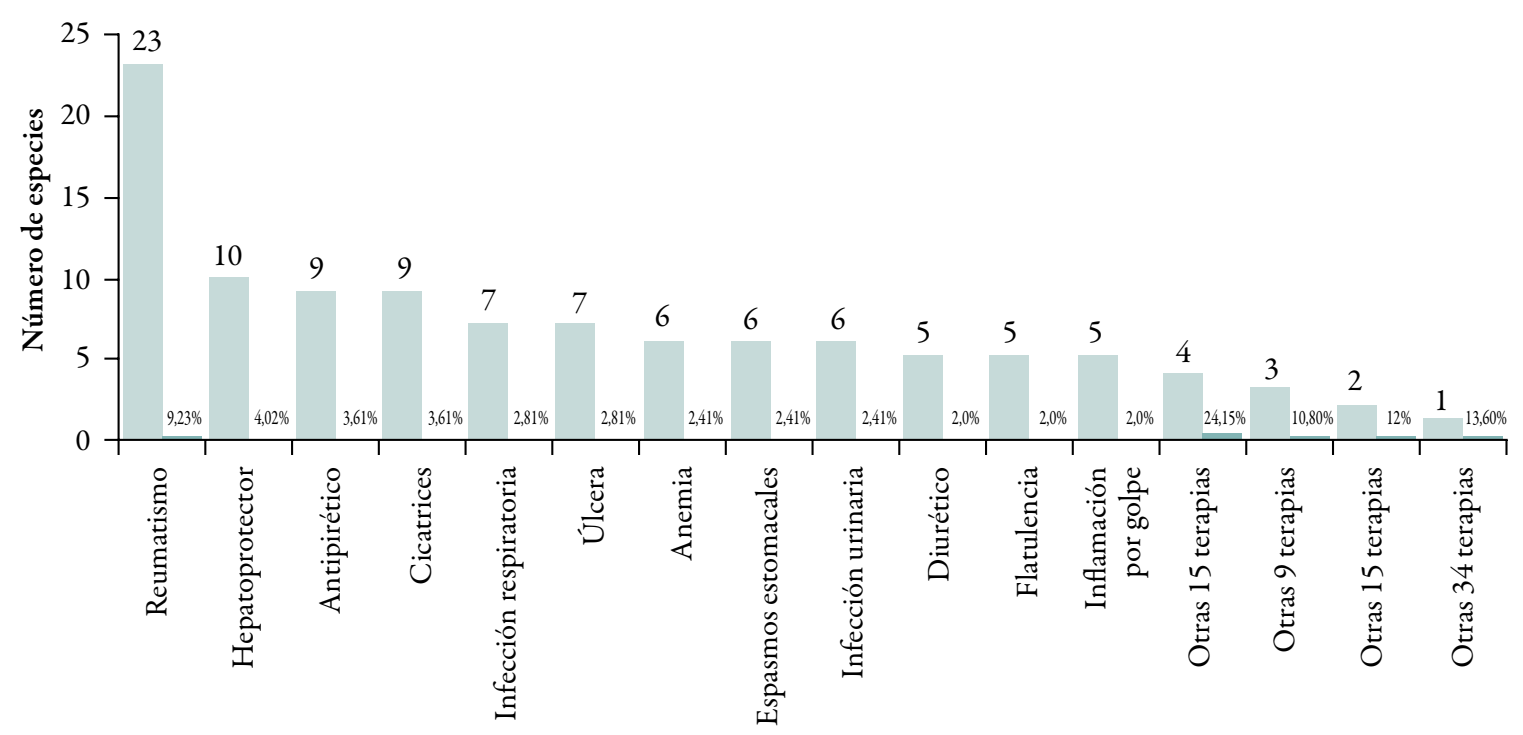

Usos terapéuticos

Fuente de información: entrevistas y encuestas a los pobladores.

Figura 2. Usos terapéuticos más frecuentemente tratados con especies medicinales de la provincia de Tambopata, Madre de Dios 


\section{Tabla 3. Partes utilizadas de las especies medicinales de la provincia de Tambopata, Madre de Dios}

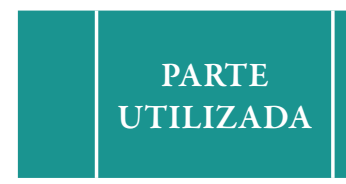

$1 \quad$ Hojas

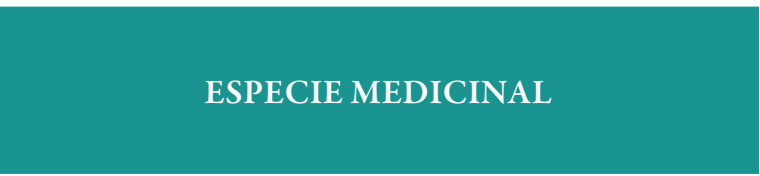

Achiachiwa, achiote, anona, arcosacha, ayapira, barbajo, barbasco, café, catahua, cetico, chanca piedra, chillca, cidra, coca, cocona, copaiba, corta corta, cuchicara, floripondio, granadilla de monte, guaco, guayaba blanca, hierba de cáncer, hierba luisa, ipururo amarrillo, kari kari macho, kinsaycucho, kirosillo, madre madre, mankapaqui, matapalo, matico mate, moco moco, montiago, oje blanco, ortiga brava, pájaro bobo, palta, pan de árbol, piquipichana, pirka, rata rata, runa manayupa, Santa María, sello sello, toronja, uña de gato, venapiticha, verbena, yanawacta, yawarchonka, yuca rosada, zarzaparrilla.

\section{No DE ESPECIES \\ POR PARTE \\ UTILIZADA}

54
$43,8 \%$

\section{PORCENTAJE \\ $\%$}

2 Tallo Anona, caña caña, chanca piedra, diente de león, jergón sacha, matico mate, moco moco, pájaro bobo, rabo del mono, rata rata, sano sano, sello sello, tamishi,

3 Toda la planta Anastasia, callampa, chanca piedra, cuchicara, guaco, hierba de cáncer, kinsaycucho, kosmello, mora, pirka, soltaquesolta, ucuchachupa, verbena.

\begin{tabular}{|c|llcc}
\hline 4 & Fruto & $\begin{array}{l}\text { Anona, café, cidra, cocona, guayaba blanca, kari kari } \\
\text { macho, palta, pan de árbol, papaya, plátano, toronja. }\end{array}$ & 11 & $9,09 \%$ \\
\hline 5 & Resina & $\begin{array}{l}\text { Catahua, cetico, matapalo, copaiba, oje blanco, paca, } \\
\text { pan de árbol, plátano, sangre de grado. }\end{array}$ & 11 & $9,09 \%$ \\
\hline 6 & Raíz & $\begin{array}{l}\text { Diente de león, solimán, uña de gato, yuca rosada, } \\
\text { chanca piedra, guaco. }\end{array}$ & 6 & $4,96 \%$ \\
\hline 7 & Corteza & Cedro, palo santo, uña de gato, piquipichana. & 4 & $3,31 \%$ \\
\hline 8 & Flor & Anastasia, arcosacha, pirka. & 3 & $2,48 \%$ \\
\hline 9 & Semillas & Achiote, palta, pan de árbol. & 3 & $2,48 \%$ \\
\hline 10 & Rizoma & Jengibre, jergón. & 2 & $1,65 \%$ \\
\hline
\end{tabular}

Fuente de información: entrevistas y encuestas a los pobladores de Tambopata, Madre de Dios. 


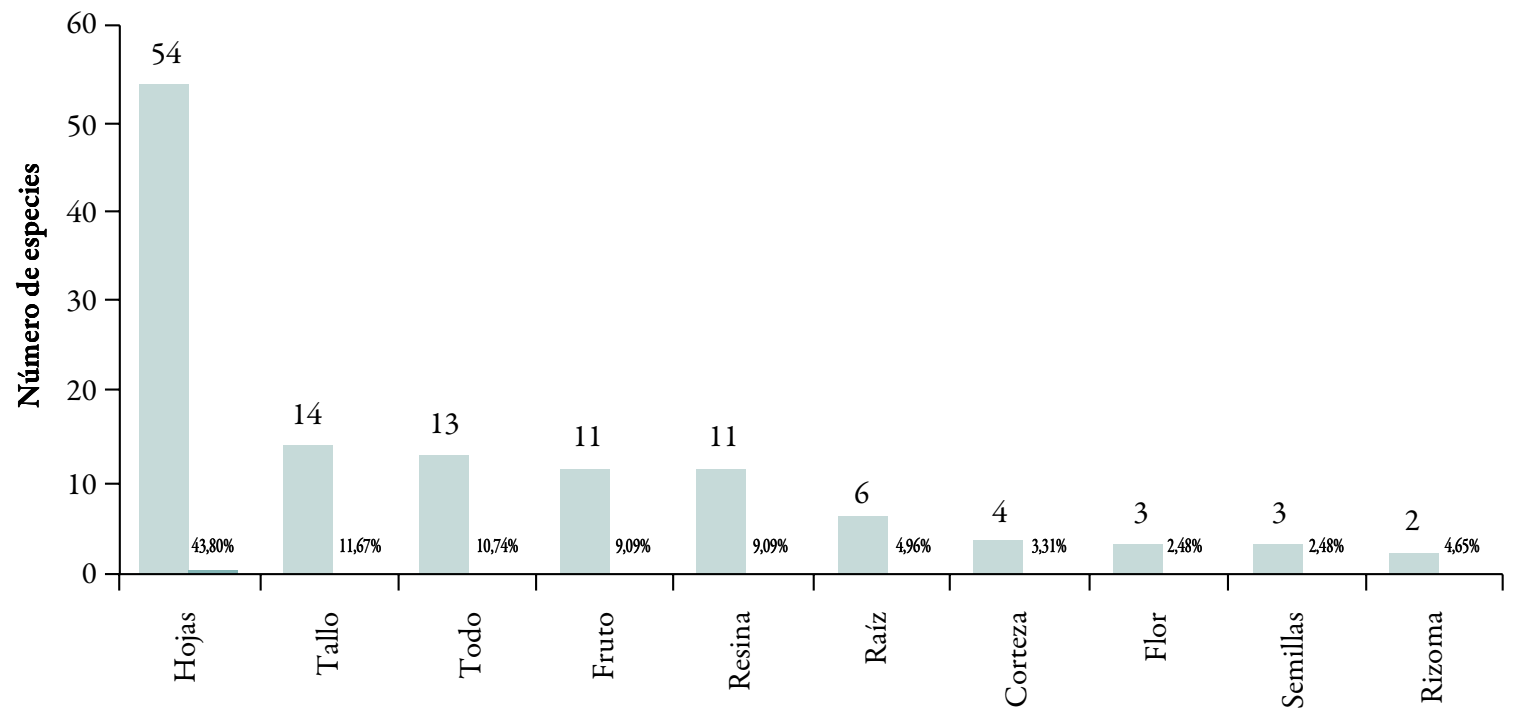

Parte de la planta utilizada terapéuticamente

Fuente de información: entrevistas y encuestas a los pobladores de Tambopata - Madre de Dios.

Figura 3. Partes utilizadas de las especies medicinales de la provincia de Tambopata, Madre de Dios.

Tabla 4. Formas de preparación de las especies medicinales en la provincia de Tambopata, Madre de Dios

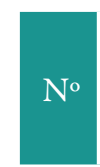

FORMA DE USO

1 Infusión

\section{ESPECIES MEDICINALES}

No DE ESPECIES POR FORMAS DE PREPARACIÓN

$23,67 \%$

Achiachiwa, achiote, anastasia, anona, ayapira, café, callampa, cedro, chanca piedra, chillca, cidra, coca, copaiba, corta corta, cuchicara, guayaba blanca, hierba de cáncer, ipururo amarrillo, kari kari, kinsaycucho, mankapaqui, matico mate, montiago, mora, no me toques, oje blanco, ortiga brava, pájaro bobo, palta, pan de árbol, piquipichana, rabo del mono, runa manayupa, Santa María, soltaquesolta, toronja, verbena, venapiticha, yawarchonka, zarzaparrilla.

2 Cocción Achiachiwa, achiote, jengibre, cedro, cidra, cocona, diente de león, guaco, guayaba blanca, hierba de cáncer, hierba luisa, jergón sacha, kirosillo, matapalo, matico mate, moco moco, ortiga brava, pájaro bobo, palo santo, pan de árbol, pirka, plátano, tamishi, uña de gato, yausapancho, yuca rosada.

3 Emplasto Achiachiwa, achiote, anastasia, ayapira, catahua, chanca piedra, chillca, coca, cocona, copaiba, hierba luisa, ipururo amarrillo, jergón sacha, kari kari, kinsaycucho, matapalo, oje blanco, pan de árbol, pirka, sangre de grado, sano sano, Santa María, solimán, ucuchachupa, yawarchonka, yuca rosada. 


\begin{tabular}{|c|c|c|c|c|}
\hline 4 & Baños & $\begin{array}{l}\text { Achiachiwa, achiote, arcosacha, barbajo, barbasco, } \\
\text { caña caña, granadilla de monte, guaco, kirosillo, } \\
\text { kosmello, madre madre, matapalo, matico mate, moco } \\
\text { moco, montiago, palta, piquipichana, rata rata, sello } \\
\text { sello, solimán, ucuchachupa, yanawacta. }\end{array}$ & 22 & $13,02 \%$ \\
\hline 5 & Zumo & $\begin{array}{l}\text { Anastasia, arcosacha, cetico, cocona, cuchicara, diente } \\
\text { de león, floripondio rosado, guayaba blanca, jengibre, } \\
\text { jergón sacha, matico mate, mora, yanawacta. }\end{array}$ & 13 & $7,69 \%$ \\
\hline 6 & Comible & $\begin{array}{l}\text { Anona, cocona, guayaba blanca, kari kari, pan de } \\
\text { árbol, sano sano, toronja. }\end{array}$ & 7 & $4,14 \%$ \\
\hline 7 & Bebible & $\begin{array}{l}\text { Caña caña, copaiba, oje blanco, paca, papaya, plátano, } \\
\text { sangre de grado. }\end{array}$ & 7 & $4,14 \%$ \\
\hline 8 & Macerado & $\begin{array}{l}\text { Barbajo, catahua, guaco, jengibre, matico mate, uña de } \\
\text { gato. }\end{array}$ & 6 & $3,55 \%$ \\
\hline 9 & Masticable & $\begin{array}{l}\text { Anastasia, coca, guayaba blanca, pájaro bobo, pirka, } \\
\text { tamishi. }\end{array}$ & 6 & $3,55 \%$ \\
\hline 10 & Compresa & Callampa, catahua, piquipichana, rata rata. & 4 & $2,37 \%$ \\
\hline 11 & Tópico & Achiote, anona, cetico, paca & 4 & $2,37 \%$ \\
\hline 12 & Tostado & Achiachiwa, café, palta. & 3 & $1,78 \%$ \\
\hline 13 & Jarabe & Anastasia, jengibre. & 2 & $1,18 \%$ \\
\hline 14 & Inhalación & Catahua, mata palo. & 2 & $1,18 \%$ \\
\hline \multirow[t]{2}{*}{15} & Parche & Matapalo & 1 & $0,59 \%$ \\
\hline & & & 169 & $100 \%$ \\
\hline
\end{tabular}

Fuente de información: entrevistas y encuestas a los pobladores de Tambopata, Madre de Dios.

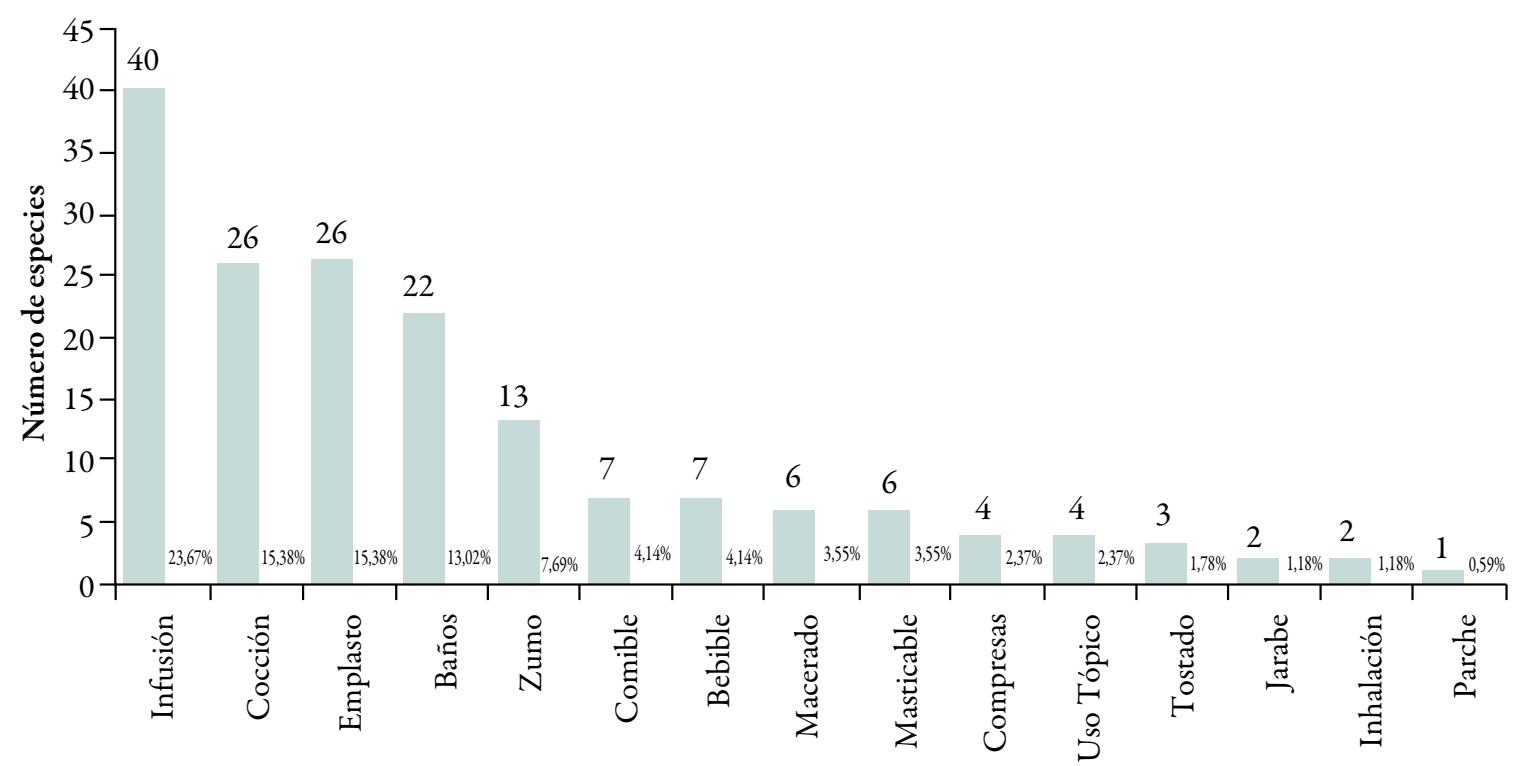

Forma de uso terapéutico

Fuente de información: entrevistas y encuestas a los pobladores de Tambopata.

Figura 4. Forma de preparación de las especies medicinales de la provincia de Tambopata, Madre de Dios 


\section{RESULTADOS}

En la tabla 1 podemos observar todos los usos posibles que se le otorga a cada una de las 75 plantas incluidas en el trabajo, siendo el Spondias mombin L. (ubus) a la que le atribuyen más usos, seguida del Piper aduncum L. (matico), Zingiber officinale (jengibre), Artocarpus altilis (Parkinson) Fosberg (pan de árbol), Croton cf. lechleri Mull Arg (sangre de grado) y Sida rhombifolia L. (ucuchachupa). En la actualidad no existe mucha información sobre Spondias mombin L. (ubus).

A partir de la tabla 1 se obtiene información importante que permitió desarrollar la tabla 2 . En la tabla 2 y figura 2, observamos 85 usos terapéuticos en enfermedades o afecciones que pueden ser tratadas con alguna(s) de las 75 especies descritas. Así tenemos que para el malestar del reumatismo utilizan un total de $9,23 \%$ de todas las plantas, lo que equivale a 23 especies, y para el hígado utilizan 10 especies de plantas. Además, podemos decir que 15 diferentes usos terapéuticos se realizan con dos especies medicinales, en tanto que 34 diferentes usos terapéuticos se valen exclusivamente de una sola planta.

Con esto podemos decir que cada comunero conoce y utiliza las plantas adecuadamente para sus diferentes afecciones, basados en su experiencia y confiando plenamente en el poder curativo de esas especies medicinales.

Los usos terapéuticos principalmente mencionados por los pobladores de Tambopata, Madre de Dios, son los malestares reumáticos y hepáticos, males que pueden explicarse por el esfuerzo físico desarrollado por varones y mujeres en sus labores de campo, así como por sus costumbres alimenticias. Respecto de su uso como antipiréticos, este se da sobre todo en los niños, por hallarse expuestos a varios agentes causantes de fiebre como insectos, basura, clima húmedo, etc.
En la tabla 3 y figura 3, observamos una distribución de 10 partes diferentes de las plantas utilizadas tradicionalmente. De las 75 muestras recolectadas, el 43,80\% de ellas, equivalente a 54 especies, aporta sus hojas, seguido por un $11,67 \%$ que ofrece sus tallos. Solo 13 plantas, la mayoría de ellas incluida en la categoría de hierbas, son utilizadas en su integridad.

En la tabla 4 y figura 4 , se puede observar que existen 15 diferentes formas de uso terapéutico de las plantas conocidas tradicionalmente. Así, podemos notar que el $23,67 \%$ de las especies, equivalente a 40 especies, se utiliza como infusión; 26 especies se utilizan en forma de cocción o emplasto, y el resto de especies, en otras formas.

\section{REFERENCIAS BIBLIOGRÁFICAS}

1. Baca Calderón, Deyvis; Ramírez OrdÓñez, Herbel. [Tesis de pregrado.] Estudio etnobotánico y etnofarmacológico de especies vegetales de interés medicinal $y$ análisis fitoquimico cualitativo de las especies más representativas de la comunidad nativa de Santa Rosa de Huacharía, distrito de Kósñipata, Cusco. Universidad Nacional San Antonio Abad del Cusco, Perú, 2008.

2. Bermúdez Arteaga, Salvador. Historia del oro de Marcapata, Quincemil y Madre de Dios. Lima, 2008.

3. Brack Egg, Antonio. Diccionario enciclopédico de plantas del Perú. Editorial del Centro Bartolome de las Casas, Cusco, 1999.

4. Gentry Alwyn H. An Overview of the Distribution and Evolution of Climbing Plants. The Biology of Vines. Printed in Great Britain at the University Press, Crambridge. 1991.

5. INEI. Censo Nacional 2007. Departamento de Madre de Dios. Tomo I, 2008. 
6. Primack, Richard. Essentials of Conservation Biology. Third Edición, Sinauer Associated, USA, 2002, p. 433.

7. Quijandría Acosta, Gabriel. Estudio etnobotánico en las cuencas altas de los rios Tambopata e Inambari. Proyecto "Gestión del Sistema de las Cuencas Tambopata Inambari y Conservación Ambiental para el Desarrollo Alternativo Sostenible en la Selva Alta y el Área Meridional de la Reserva Tambopata Candamo (ZRTC)", Cusco, 2007.

\section{Páginas web}

8. Abreu Guirado, Orlando A; Cuéllar Cuéllar, Armando. "Estrategias en la selección de las plantas medicinales a investigar". Rev. Cubana de Plantas Medicinales (jul.-sept. 2008); 13(3). Disponible en:

http://bases.bireme.br/cgi-bin/wxislind. exe/iah/online/?IsisScript=iah/iah. xis\&src

9. OMS. "Estrategias de la OMS sobre medicina tradicional”. Geneva, 2002.

Disponible en:

http://www.amhb.org.br/media/ estrategia.pdf

10. Vargas Torres, Boris Ilich. "Historia de las plantas medicinales". Revista Mundo Natural. España, 2005.

Disponible en:

http://www.biomanantial.com/historiade-las-plantas-medicinales-a-87.html 


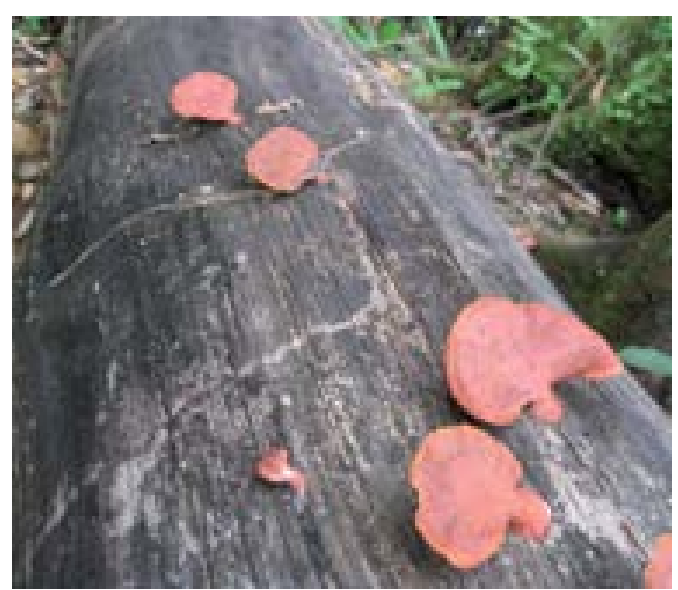

Callampa (Pycnopons cf. sanguineus L.)

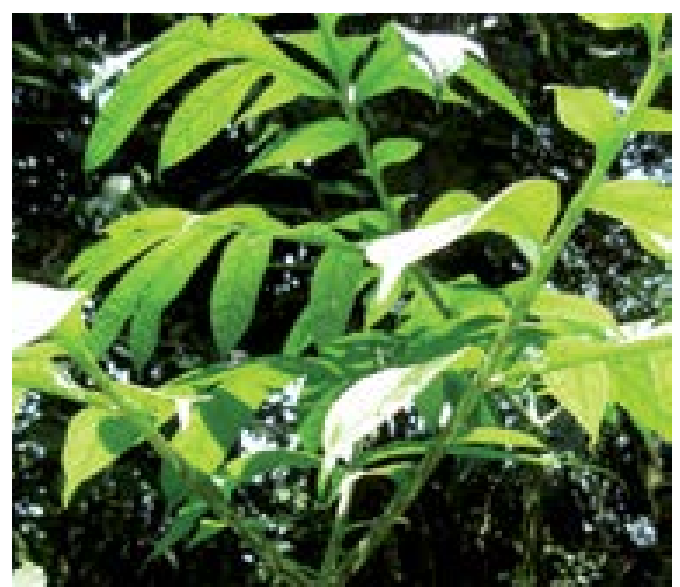

Jergon sacha (Dracontium plowmanii)

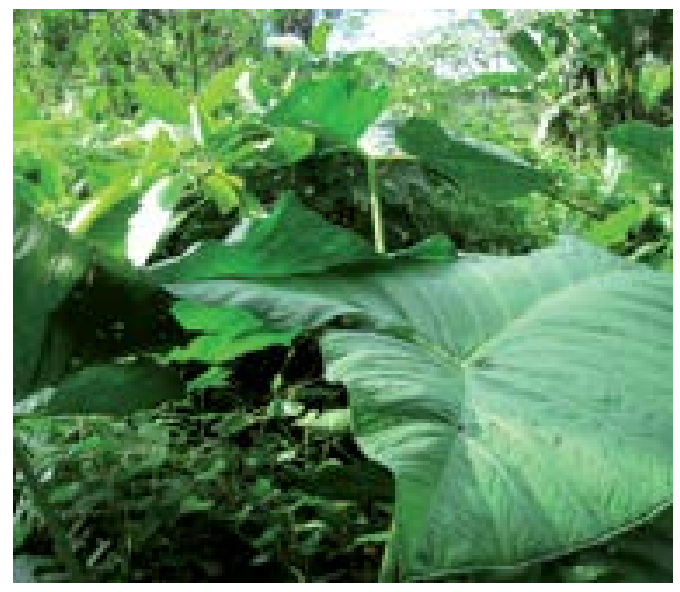

Soliman (Lobelia decurrens Cav)

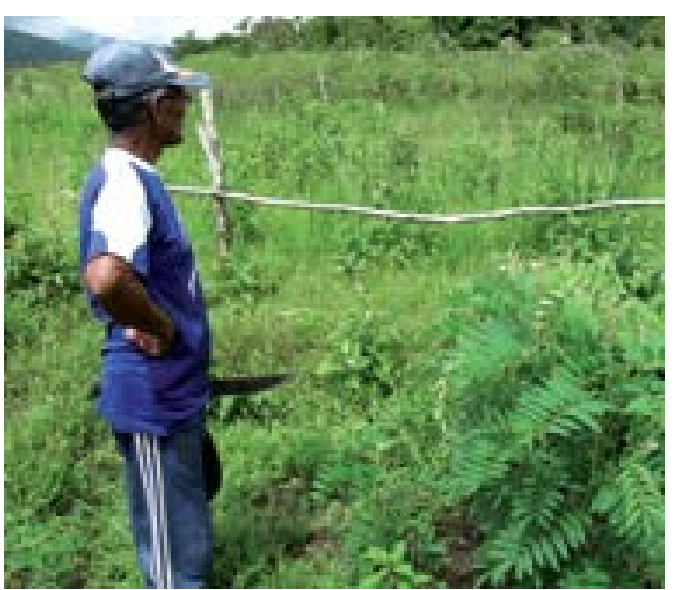

Curandero de Tambopata

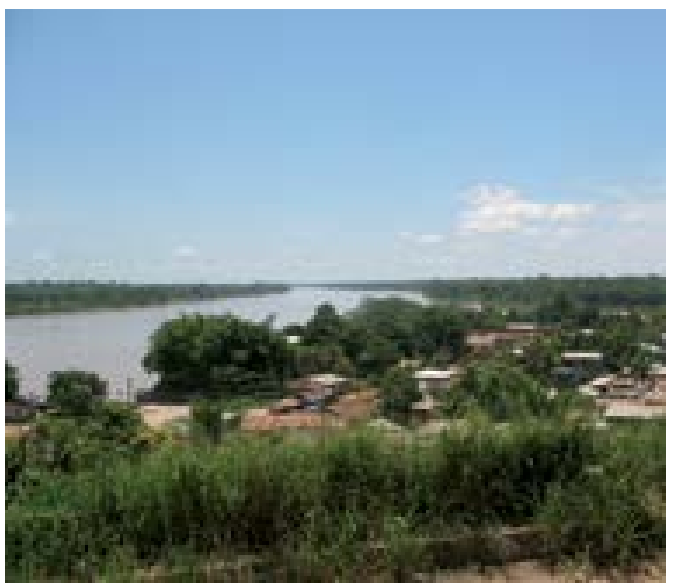

Río Tambopata

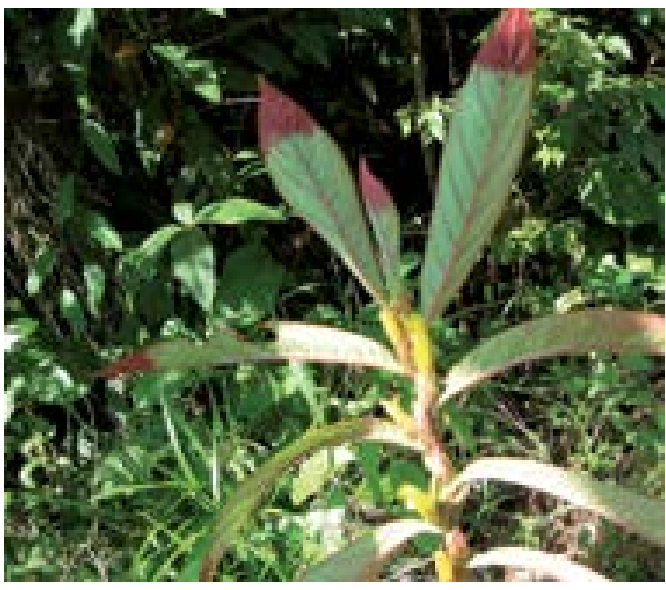

Yawarchonka (Columnea ericae Mansf.) 


\section{ENTREVISTA SOBRE LA UTILIZACIÓN DE PLANTAS MEDICINALES.}

Entrevista \#:

Nombre del entrevistado:

Lugar de Nacimiento:

Nombre común de la Planta:

\section{DATOS GENERALES}

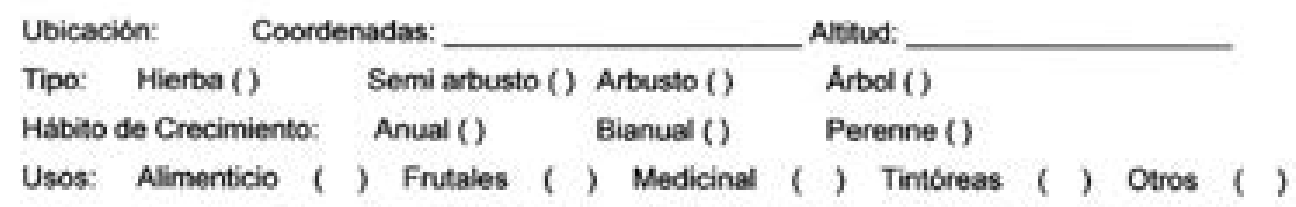

¿Para qué enfermedades usa la planta?

a)

b)

c)

Como usa (Forma de uso)

a) Emplastos () Bahios () Macerados () Infusión () Decocción () Otros ( )

b) Emplastos () Bafios () Macerados () infusión () Decocción () Otros ( )

c) Emplastos () Bafios () Macerados () Infusión () Decocción () Otros ( )

¿Qué parte de la planta utilizas?
a) $\operatorname{Raiz}$ ()
Tallo () Hojas ()
Flores ()
Fruto () Otros ()
b) Raiz ()
Tallo () Hojas ()
Flores ()
Fruto () Otros ()
c) Raiz ()
Tallo () Hojas ()
Flores ( )
Fruto () Otros ()

¿Con qué frecuencia las utiliza? (Dosis)

a) Una sola vez () Cuantas vez por dia/cuantos dias ( / ) Otras

b) Una sola vez () Cuantas vez por dia/cuantos dias ( $f$ ) Otras

c) Una sola vez () Cuantas vez por dialcuantos dias ( $t$ ) Otras

¿Esta planta le genera efectos indeseables?

¿Mezclas esta planta con otras plantas? Si () No (), ¿Cuales?
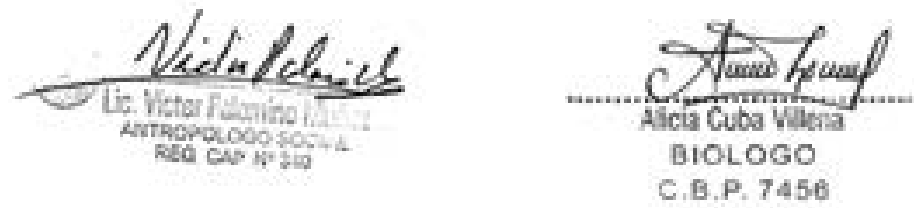
ANEXO No 02

ENCUESTA SOBRE EL CONOCIMIENTO DE PLANTAS MEDICINALES.

Encuesta \#:

Fecha:

Entrevistador

Nombre del entrevistado:

Edad:

Lugar de Nacimiento:

Afhos que radica en Quincemil:

¿Conoce Ud. Las siguientes plantas y para que las utiliza?

\begin{tabular}{|c|c|c|}
\hline $\begin{array}{l}\text { Nombere } \\
\text { comín }\end{array}$ & SUNO & usos \\
\hline Achiachiwa & & Reumatismo, indecoion urinaria \\
\hline Achiote & & Probtata quemadura, int Urinaria, regelente \\
\hline Ajengiber & & Diurva, colleses, vomitos, restrio, neumatismo, conjurtivetis \\
\hline $\begin{array}{l}\text { Anastasia, } \\
\text { Botoncillo }\end{array}$ & & 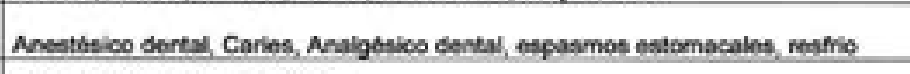 \\
\hline Anona & & Cancee, Raquitivmo, Dabetes \\
\hline Arcosacha & & Micosis, Contusiones, cicatrices. \\
\hline Ayapira & & Reumatismo, Cicatrices \\
\hline Barbajo & & Cicatrices profundas. \\
\hline Cate & & Cardictorico, gases, parto doloroso, \\
\hline Callampa & & Hemorregla post parto, hemorrogia oulmonar, Úloera, Heridas sangrantes. \\
\hline Caha canta & & Hepatoprotecter, febre smurile presion ata. \\
\hline Catahua & & tumores, turidas, vomitos abeceso \\
\hline Cediro & & Fiebce, tos, diarrea. \\
\hline Cetico & & picaduras de insectos, hemorragia \\
\hline $\begin{array}{l}\text { Chanca } \\
\text { Piedra }\end{array}$ & & Resumusisma, Higeda, Calculos de la vesicula, Clcatrisantle, produccion de leche. \\
\hline Chisica & & Reumatsmo, fracturas \\
\hline Cidra & & Flatulencias dolones menstruales, colerina, infectiones nespiratorias. \\
\hline Coea & & Dolor estomacal picaduras de irsectos. los. \\
\hline Cosona & & Ulocera, Diabeles, Hepatitis, Picaduras de insectos \\
\hline Copaiba & & Purfica la sangre, hemoeragia, quemadura. \\
\hline Corta Corta & & Hemorragia. \\
\hline Cuchicara & & Higado, diemeas, estretimiento, emoucho, ardor de pie, depurativo. \\
\hline $\begin{array}{l}\text { Diente de } \\
\text { Leon }\end{array}$ & & Diabetes, Heqatopectector \\
\hline $\begin{array}{l}\text { Floripondio } \\
\text { Rosado }\end{array}$ & & Mondedura de sergiente. \\
\hline $\begin{array}{l}\text { Granadilia de } \\
\text { monte }\end{array}$ & & Fiebre \\
\hline Guaco & & Reumatiemo, inflamaden taringea. \\
\hline $\begin{array}{l}\text { Guayaba } \\
\text { Blanca }\end{array}$ & & Flatulencia, hepetsocotector, calembres, conjuntevitis estrenimiento, odontabias. \\
\hline $\begin{array}{l}\text { Hierba do } \\
\text { Cancer }\end{array}$ & & Cicatrisante, cincer, infecolones, dianteico. \\
\hline Hierba Luisa & & Infocciones reaspiratorias, Digestivo \\
\hline $\begin{array}{l}\text { Ipururo } \\
\text { Amarrillo }\end{array}$ & & Disenteria, herpes, reumatiemo, collicos estomacales. \\
\hline Jergon sacha & & Mondedura de serpiente, hemia, Úloera, parkinson \\
\hline kari kari & & Inflansodion por golges tos. \\
\hline Kinsaycucho & & Inflamecion, Reumutsmo. \\
\hline Madre madre & & Dolor de huesos. \\
\hline
\end{tabular}
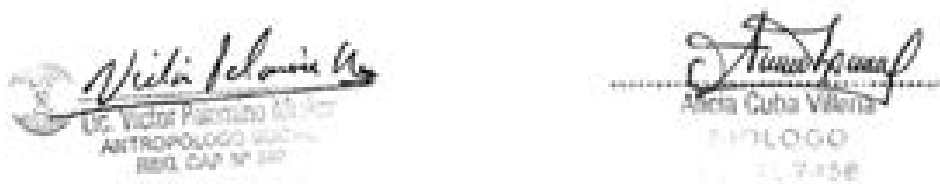